\title{
PLASTIC INSTABILITY IN COPPER AND BRASS AND ITS RELATION TO MICROSTRUCTURE AND TEXTURE
}

\author{
T. Leffers and V.S. Ananthan \\ Materials Department, Risø National Laboratory, DK-4000 Roskilde, Denmark
}

\begin{abstract}
Second-generation microbands in copper and bundles in brass reflect plastic instabilities. The possible crystallographic rules for the selection of grains with such instabilities are investigated. In copper the existence of simple crystallographic rules is questionable. In brass there seems to be a set of crystallographic rules which is in reasonable agreement with theoretical considerations.
\end{abstract}

\section{INTRODUCTION}

The microbands ${ }^{1,2}$ in rolled copper - or second-generation microbands as we suggest to call them in order to avoid confusion with "first-generation microbands" (refs. ${ }^{3,4,5}$ ) - reflect a plastic instability. They are plate-shaped zones parallel to $\{111\}$ with shear far greater than that in the surrounding material. The "bundles" ${ }^{3,6}$ in rolled brass, consisting of a composite of matrix and thin lamellae of deformation twins, reflect a similar plastic instability. Bundles are also observed in austenitic steels. ${ }^{6,7,8}$ Such instabilities seem to be absent in f.c.c. metals with higher stacking fault energy (aluminium and nickel), but second-generation microbands are observed in $\mathrm{Al}-4.8 \% \mathrm{Mg}$. ${ }^{9}$ It is an obvious question whether the second-generation microbands and the bundles are two completely different phenomena or whether they are related (via the relatively low stacking fault energy in the materials in which they form) in spite of the microstructural differences.

In this work we present a detailed investigation of the orientation of the grains in which second-generation microbands and bundles form - in order to see whether there are crystallographic similarities between the two types of plastic instability (apart from their common orientation parallel to $\{111\}$ ).

\section{MATERIALS}

The materials investigated are copper and brass (with $15 \%$ zinc by weight), both of $99.95 \%$ purity. The cast materials were subjected to a dual recrystallization (consisting of rolling to moderate reduction followed by recrystallization at $550^{\circ} \mathrm{C}$ in helium atmosphere). This resulted in almost texture-free materials with grain size $100 \mu \mathrm{m}$ and $40 \mu \mathrm{m}$ for copper and brass, respectively. These starting materials were rolled to reductions of $28 \%$ for copper and $26 \%$ for brass. At such reductions the microstructure 


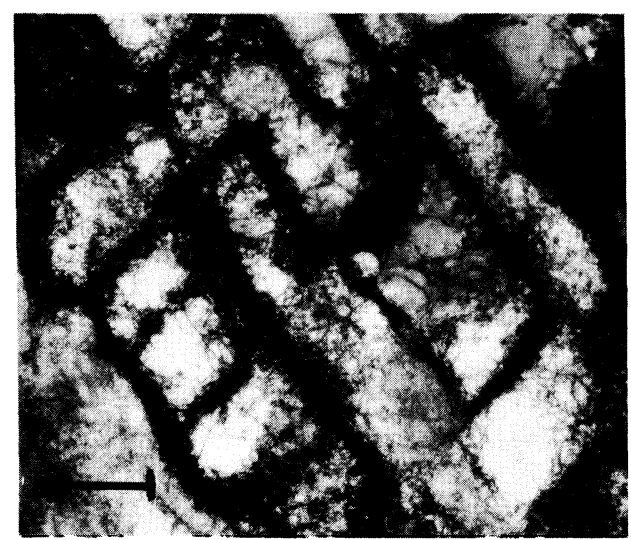

Fig. 1. HWD structure in copper.

The line shown is $0.5 \mu \mathrm{m}$ long.

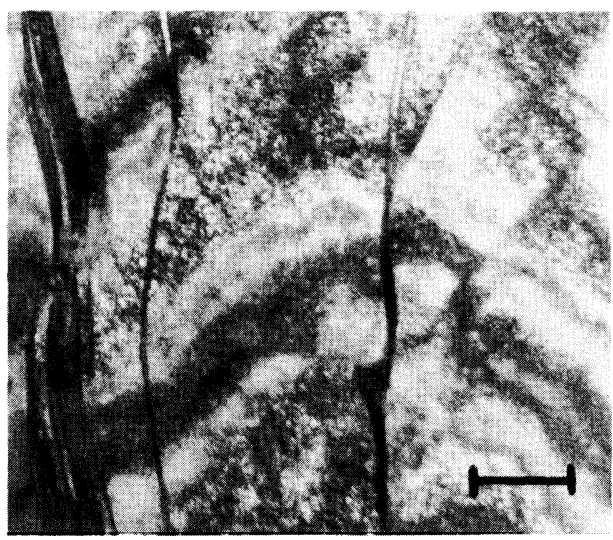

Fig. 2. Scattered twins in brass. The line shown is $0.5 \mu \mathrm{m}$ long.

is well developed, and the texture is not too sharp. The latter is important because it means that there are still a wide range of orientations available for the investigation of the orientation dependence of the tendency to form s-g microbands and bundles.

\section{EXPERIMENTAL PROCEDURES}

Disc-type thin foils for transmission electron microscopy were made by jet polishing in an electrolyte with $25 \%$ phosphoric acid and $25 \%$ ethylene glycol in water. The foils were examined in an electron microscope operating at $200 \mathrm{kV}$.

At moderate reductions there are two types of grains in copper ${ }^{4}$ : grains with a high density of dislocation walls which subdivide the grains in parallelogram-shaped areas as shown in fig. 1(HWD grains), and grains with a low density of dislocation walls (LWD grains) which are characterized by equiaxed cells. LWD grains practically always contain second-generation microbands at the reduction we deal with, whereas these microbands are rare in HWD grains. In order to facilitate the investigation we characterize the grains by their type of structure (HWD or LWD) which is very easily observed and not directly by the frequency of second-generation microbands: we monitor the orientation and the type of structure for a number of randomly selected grains.

In brass we distinguish between three types of grains: those with bundles, those without bundles and twins and, as an intermediate type, those with scattered twin lamellae as shown in fig. 2. Again we monitor the orientation (of the matrix, not the bundles or twins) and the type of structure for a number of randomly selected grains.

\section{RESULTS}

The results are plotted in inverse pole figures as shown in figs. 3 and 4. The overall distribution of points (disregarding their type) is in approximate agreement with the textures of the two materials which indicates that the grains selected are reasonably representative for the materials. The number of grains investigated is 100 and 80 in copper and brass, respectively. 

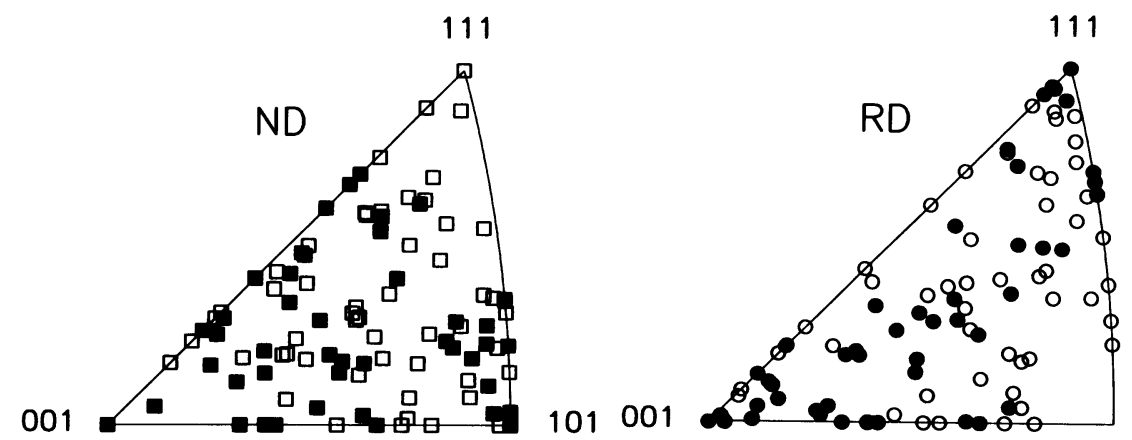

Fig. 3. Orientation distribution in copper, LWD grains are black, HWD grains are white. Thus, grains with high frequency of second-generation microbands are black.

In copper about $45 \%$ of the grains are LWD grains. There seems to be some tendency for the two types of grains to be concentrated in different orientation ranges, but the overall pattern, if it exists, is very complex. There is only one clear trend: all the observed grains with orientations near $\{110\}<001>$ have LWD structure, i.e. a high frequency of second-generation microbands.

In brass the fraction of grains with bundles is about 35\%. There is a clear trend that the orientations of the three types of grains are segregated (even though the orientation ranges overlap). This implies that the grains with scattered twin lamellae form a distinct group of their own as far as their orientations are concerned. The results are in agreement with earlier semiquantitative investigations ${ }^{3,6,10}$ showing that there are few or no twins in grains with $\{110\}$ approximately parallel to the rolling plane. As a matter of fact our observations show that there are no twins in grains with orientations close to $\{110\}<001>$ whereas there are scattered twins in many grains with orientations close to $\{110\}<112>$.

As to the idea that there may be a relation between the orientations of the grains with second-generation microbands in copper (the LWD grains) and the grains with bundles in brass, the orientation distributions in figs. 3 and 4 show no obvious relation. On the contrary, the only clear trend for orientation segregation in copper (many

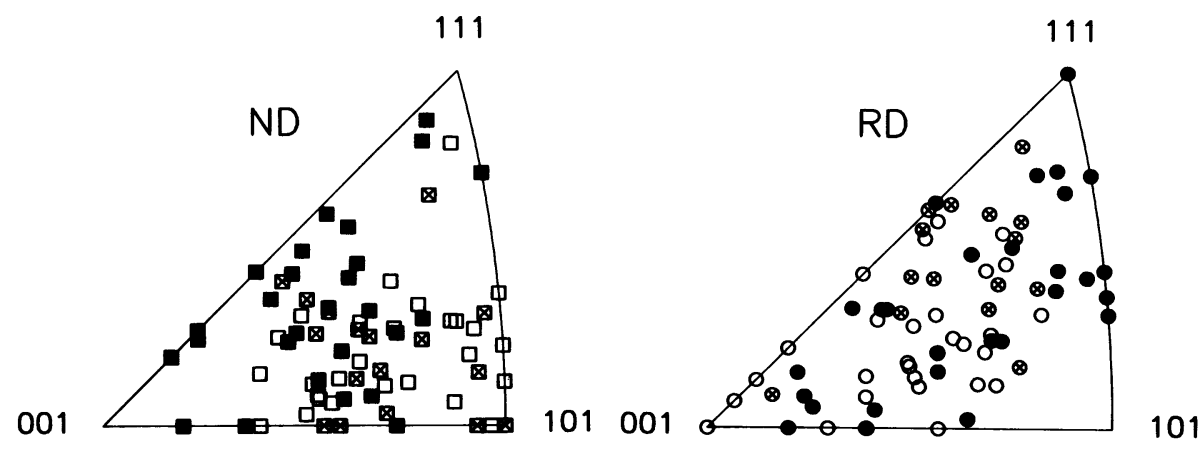

Fig. 4. Orientation distribution in brass. Grains with bundles are black, grains without twins are white, grains with scattered twins are white with cross. 


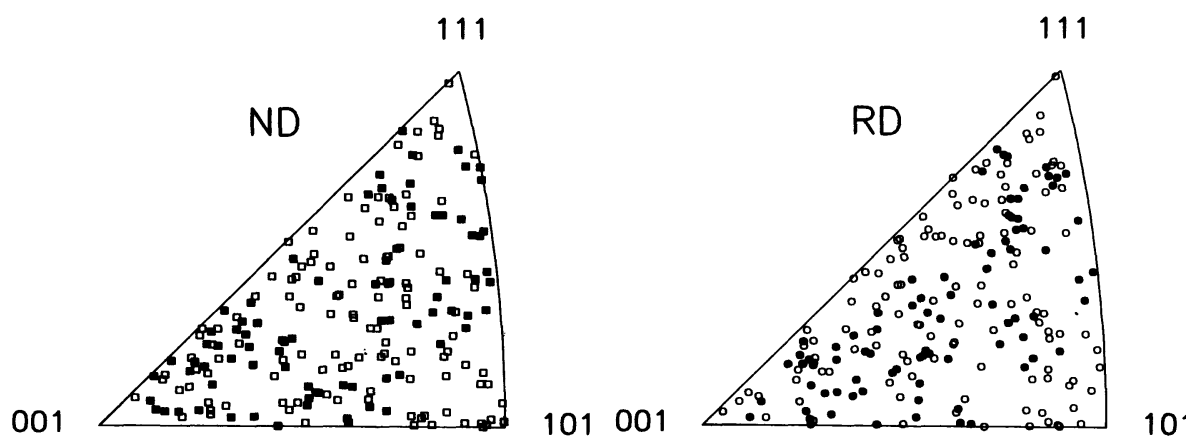

Fig. 5. Distribution according to the ratio between the two highest resolved shear stresses; black $\sim$ ratio $>1.47$, white $\sim$ ratio $\leq 1.47$.

microbands in grains with orientations near $\{110\}<001>$ ) is directly opposite to the situation in brass where these orientations have no bundles.

\section{CALCULATED DISTRIBUTIONS}

If one should hope to see any relation between the orientations of the grains with many second-generation microbands in copper (the LDW grains) and the grains with bundles in brass, this would have to be based on some crystallographic rule which is not related to twinning. It has been suggested ${ }^{1,3}$ that the second-generation microbands may represent a concentration of slip on one selected slip plane. The bundles certainly represent a concentration of slip on one slip plane. ${ }^{3,6}$ Therefore we have grouped 200 randomly oriented grains according to the ratio between the resolved shear stresses (from a Tucker-type applied stress ${ }^{11}$ ) for the most heavily loaded and the next most heavily loaded slip systems. A selected limit for this ratio determines which group a specific grain belongs to (the group with lower or the group with higher values of the ratio). By selecting a limit of 1.47 we obtain that $40 \%$ of the grains come in the group with higher values which approximately corresponds to the fractions of LWD grains in copper and grains with bundles in brass. The resulting distribution is shown in fig. 5 . We have made the same exercise for two lots of $\mathbf{2 0 0}$ grains with orientation distributions representative of the textures in the two materials investigated, but the distribution of black and white points was not significantly different from that in fig. 5 .

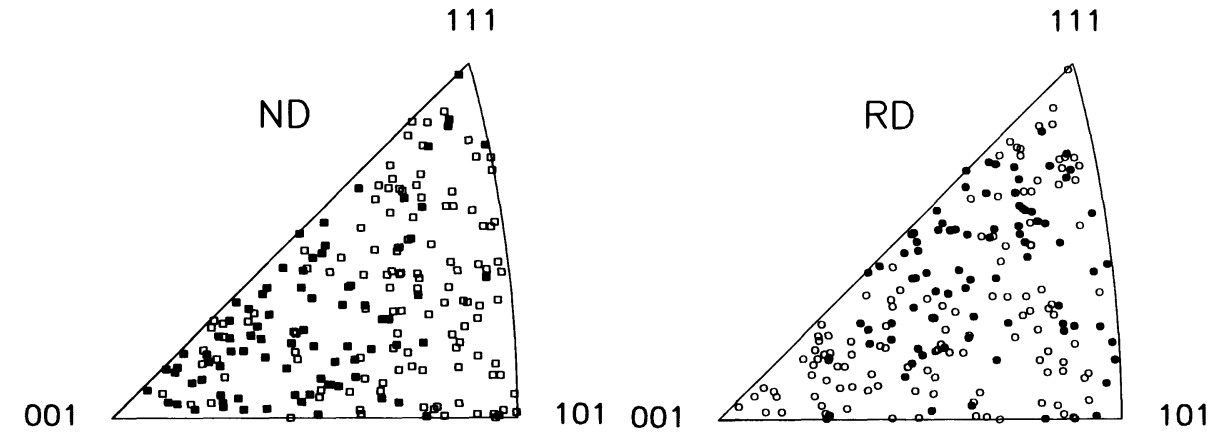

Fig. 6. Distribution according to the ratio between the highest resolved shear stresses for twinning and slip; black $\sim$ ratio $>0.95$, white $\sim$ ratio $\leq 0.95$. 
There is no obvious similarity between the observed distribution of black and white points for copper in fig. 3 and the calculated distribution in fig. 5 . There is some similarity between the observed distribution of black and white points for brass in fig. 4 and the calculated distribution in fig. 5 , but the agreement is far from perfect.

With special reference to brass we have produced calculated distributions of the type in fig. 5 with distinction according to the ratio between the highest resolved shear stresses for twinning and slip. A distribution with $40 \%$ of the grains in the group with relatively high resolved shear stress for twinning (obtained by selecting a limit for the ratio of 0.95 ) is shown in fig. 6. There are obvious similarities between the experimental distribution in fig. 4 and the calculated distribution in fig. 6, but again the agreement is not perfect. We shall return to the agreement/disagreement in the next section.

\section{DISCUSSION}

It should be underlined that we have deliberately limited our investigation to a moderate level of reduction. This implies that our observations are not necessarily representative of the materials behaviour at higher reductions.

The present work only reveals one clear crystallographic rule for the orientation dependence of the formation of the two types of structures in copper (LWD and HWD structure) and hence for the formation of second-generation microbands: grains with orientations near $\{110\}<001>$ have LWD structure and hence a high density of microbands. However, there is the possibility that replotting fig. 3 in three-dimensional Euler space may reveal a complex pattern which is not visible in the present two-dimensional plot.

Apart from a simple orientation dependence one may imagine three other principles which could determine the type of structure that a grain develops: (i) it may be determined by grain size, (ii) it may be determined by some combination of the orientation of the grain in question and the orientation of the surrounding grains, and (iii) it may depend on some self-organization scheme which produces a distribution of grains with different deformation patterns and hence different microstructures. Principle (i) has some indirect support in the work of Gracio, Fernandes and Schmitt. ${ }^{12} \mathrm{~A}$ possible grain-size effect is not so obvious that we have noticed it, but it would be reasonably simple to make a quantitative investigation. A proper investigation of principle (ii) would be very tedious. Principle (iii) would be the one we are left with if none of the others apply. With the present complete lack of understanding of the principle that governs the formation of second-generation microbands we cannot go into a discussion of their effect on texture and overall deformation pattern.

The observations on brass indicate that the formation of bundles (and scattered twin lamellae) is largely governed by the orientation - but we cannot exclude that the above alternative principles (i), (ii) and (iii) also have a role to play. Again it applies that plotting in three dimensions may reveal some more details. We suggest that the main governing principle for the formation of bundles is a combination of one principle similar to that used in fig. 5 and one similar to that used in fig. 6 . For instance one notices that the bundles formed in grains with RD <014 > agree with fig. 6 but not with fig. 5. And one notices that grains with RD $\sim 112>$ should twin according to fig. 6 , but 
they should not form bundles according to fig. 5 ; in fact they tend to form scattered twin lamellae. Some of these scattered twins are actually formed in grains with orientations close to $\{110\}<112>$ which should not twin according to fig. 6; this indicates that some modifications are needed. It should be mentioned, however, that static charts as figs. 5 and 6 cannot be expected to give a perfect description of the experimental observations in fig. 4. Fig. 4 gives the orientations of the different grains after $26 \%$ reduction and not the orientations when bundles/twins started to form. Ideally the static charts should be components in a dynamic simulation which keeps track of the orientation changes after the onset of the formation of bundles/twins.

The impact of the plastic instability in brass as represented by the bundles is clear $^{3,6}$ : it produces glide on one single slip plane in the grains with bundles, hence changing the deformation pattern and the derived texture from the Taylor-type towards the Sachs-type.

We had hoped to find a set of related crystallographic rules for the plastic instabilities in copper (as represented by the second-generation microbands) and brass (as represented by the bundles). Such related rules obviously do not exist. This does not exclude that the two types of instability are related at the level of the underlying dislocation processes, but the detection of such a possible relation would go beyond the level of sophistication that we master at present.

\section{ACKNOWLEDGEMENTS}

We want to thank N. Hansen and D. Juul Jensen for helpful discussions, J. Lindbo for preparing the specimens for electron microscopy and L. Jørgensen for typing the manuscript.

\section{REFERENCES}

1. A.S. Malin and M. Hatherly, Met. Sci. 13, 463 (1979).

2. M. Hatherly, in "Proc. ICSMA 6" (R.C. Gifkins, ed., Pergamon Press, Oxford 1982/83) p. 1181.

3. T. Leffers and N. Hansen, in "Materials Architecture" (J.B. Bilde-Sørensen et al., eds., Risø National Laboratory 1989), p. 127.

4. V.S. Ananthan, T. Leffers and N. Hansen, Mater. Sci. Techn. (in press).

5. N. Hansen, Mater. Sci. Techn. (in press).

6. T. Leffers and J.B. Bilde-Sørensen, Acta metall. mater. (in press).

7. M. Blicharski and S. Gorczyca, Met. Sci. 12, 303 (1978).

8. C. Donadille, R. Valle, P. Dervin and R. Penelle, Acta metall. 37, 1547 (1989).

9. A. Korbel and P. Martin, Acta metall. 34, 1905 (1986).

10. B.J. Duggan and C.S. Lee, in "Materials Architecture" (J.B. Bilde-Sørensen et al., eds., Risø National Laboratory 1989) p. 325.

11. G.E.G. Tucker, J. Inst. Metals 82, 655 (1953/54).

12. J.J. Gracio, J.V. Fernandes and J.H. Schmitt, Mater. Sci. Eng. A118, 97 (1989). 